\title{
Application of a Six-Step Bionic Strategy for Achieving Product Segmentation
}

\author{
Elena Angeleska, Kristijan Vasilevski, Ile Mircheski, Sofija Sidorenko \\ Ss.Cyril and Methodius University in Skopje, Faculty of Mechanical Engineering, \\ Rugjer Boshkovikj 18, Skopje, North Macedonia
}

\begin{abstract}
In this paper, the six-step strategy for bionic inspiration in industrial design and engineering is improved with involvement of an additional tool functional decomposition. The improved strategy is explained through a design case study. The case study uses a "bottom-up" approach for identifying the key functionalities of the dandelion flower which is recognized as a unique natural phenomenon. Those functionalities are then redefined in a technical manner and used for designing a solar street light with the goal to achieve unique aesthetics and segmentation (modularity). The presented tools for systematization of gathered bionic information can help make the bionic product development processes more efficient.
\end{abstract}

Keywords - Bio-inspired design, Bionic six-step strategy, Functional decomposition, Dandelion flower, Street light.

\section{Introduction}

Bionics, biomimetics, or biologically inspired design is a methodology commonly practiced by designers, architects and engineers since it provides an inexhaustible source of inspiration and adequate techniques for transferring the ideas to practical problem solutions .

DOI: 10.18421/TEM111-24

https://doi.org/10.18421/TEM111-24

Corresponding author: Elena Angeleska,

Ss.Cyril and Methodius University in Skopje, Faculty of Mechanical Engineering, Rugjer Boshkovikj 18, Skopje, North Macedonia.

Email: elena.angeleska@mf.edu.mk

Received: 02 September 2021.

Revised: 24 January 2022.

Accepted: 31 January 2022.

Published: 28 February 2022.

(c) BY-NC-ND (C) 2022 Elena Angeleska et al; published by UIKTEN. This work is licensed under the Creative Commons Attribution-NonCommercial-NoDerivs 4.0 License.

The article is published with Open Access at https://www.temjournal.com/
The success of the practical application of bionic principles lies in the multidisciplinary core of bionics, which merges the knowledge from biology together with various other sciences.

Scientist and writer Janine Benyus popularized biomimicry by publishing the book "Biomimicry: Innovation Inspired by Nature" in 1997 [1]. In her book, she explains how organisms are the best models to follow since they "have done everything we want to do, without guzzling fossil fuel, polluting the planet, or mortgaging their future" and therefore we need to find ways not to control nature, but learn from her. Since then, various design methods have emerged for this goal precisely - learning from nature. They guide engineers, designers and other professionals through the stages of seeking inspiration in natural phenomenon and reshaping the recognized shapes, patterns and functionalities of living organisms in a manner that they can be applied in the new product development process. Most influent examples of such strategies are the "bidirectional bionic design method" of Versos and Coelho [2] and the "organizing framework for practicing of biologically inspired design", proposed by Helms, Vattam and Goel [3], [4].

Designers commonly rely on these two bionic approaches when exploring the possibilities which are offered by natural systems in the context of design and engineering. They either begin by investigating natural solutions and abstracting their principles which are used as an inspiration in the design process - this being the "solution-driven" or "Top-Down" approach. The other approach is doing the reverse, analysing a recognized design problem and turning to nature to seek for a solution in biological systems - which is the "problem-driven" or "Bottom-Up" method.

Researches Stevanović and Rašuo [5] focused on a "Bottom-Up" approach to work on the development of a particular engineering solution. Their research is based on laboratory analysis of the movement of various legged natural organisms. According to the observations and measurements made, they recognized 4 types of leg movement. For those movement mechanisms, they conducted further 
mathematical analysis (by SolidWorks and Matlab Simulink) using a generated 3D CAD model of a robot resembling an insect with 6 legs. They successfully prove the point that innovation in engineering is highly possible by joining bionic inspiration with contemporary engineering tools and software.

Filippov, Gorb, and Popov analyze the mechanical and chemical mechanisms of tardigrades and how their characteristics can help to develop biologically motivated artificial adhesive films and robotic systems [6].

Aside from these two main strategies, there are plenty of other bionic design approaches available in literature [7]. Modern approaches of bionic methods are based on systematic analysis and functional decompositions. Santos-Reyes and Lawlor-Wright propose developing structural models of the manmade systems that need to be improved. Structural models help to understand all the system parts and their relations and pinpoint characteristics that need to be changed by using functionalities from a recognized natural system [8]. Similarly, López Forniés and Berges Muro suggest conducting a functional analysis of a system (product), then doing the biological research, translating the found solutions into key functions, and using them for product concepts [9].

The strategy elaborated in this paper includes steps for both (1) responding to a design problem with a nature-inspired solution and (2) finding an adequate application of a recognized unique natural phenomenon. Functional decomposition processes lie in the core of both design flows because they help determine key functions of product systems and natural systems. This is a starting point for redefining the bionic functions in a manner that they can be used to solve product functions. The proposed strategy provides a way to collect the transformed bionic solutions as morphological matrixes of known solutions, or principles, inspired from nature that can be used to solve product contradictions and be an inspiration for creating product innovations.

\section{Design Case Study}

The proposed strategy for bionic inspiration is explained through an example of a project used as a case study. The project follows the "Bottom-Up" flow and explains how the functionalities and appearance of a particular product are improved by applying bionic inspiration in the design process. This section of the paper firstly explains the natural phenomenon which was identified and used and then elaborates its' functional decomposition and the six-steps strategy for reaching the concept generation phase.

\section{A. Bionic Inspiration}

The dandelion is a perennial weed commonly found in Europe and North America. Its scientific name is "Taraxacum officinale" and it is a member of the "Compositae" family. What is characteristic about it is that the flower is actually composed of many florets, and not one individual flower (Figure 1a). The florets are located at the top of the stem and they are protected by so-called involucre. Involucre are the green leaves surrounding the florets for their support and protection from predators (Figure 1b). What is interesting about the florets is that each of them is composed of all the essential flower parts (Figure 1c). The individual florets have yellow petals that attract pollinators. As we move toward the stem, these petals narrow into a tube that provides nectar and lies on an ovary with a single ovule. As the florets mature, the stigma grows through the tube and splits into two curling lobes covered by pollen. When fertilized by pollen the ovule becomes a seed. The seeds have a tip shaped like a beak for attachment to the spherical head of the flower. This attachment is possible because the head has a shape with tiny holes that follow a symmetrical pattern. Between the ovary and the petal there is tuft of white fibers, or hairs, called the pappus. These become a white ball later on and serve the purpose of dispersing the seed by flying in the wind with a parachute-like system (Figure 1d).

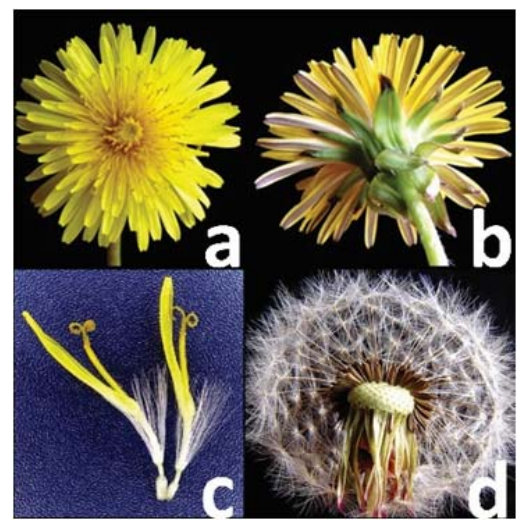

Figure 1. a) Dandelion flower composed of individual florets; b) Dandelion involucre; c) Single florets composed of petals, nectar tube, stigma, ovary and pappus;

d) Detachment of the ripe seeds from the flower head by the pappus (source of images: Brian Johnston, A Close-up View of the Wildflower "Dandelion", microscopyuk.org.uk/, 2010)

The easy detachment of the seeds by the lightest breeze is possible due to the design of the holes on the head section and their difference in diameters which make an ideal tight, but not too tight opening that holds the beak of the seed in position, but also allows the seed to be flown away when ripe. After being detached, the drag-enhancing pappus is what helps to keep seeds aloft. The complexity, beauty, 
functionality and composition of the dandelion flower have been used as a bionic inspiration in many researches so far [10], [11]. This paper explains how using the proposed bionic methodology helped to draw inspiration from the dandelion in order to create a product with unique aesthetics and modular design for simplified manufacture, repair and adaptability.

\section{B. Functional Decomposition}

Functional analysis serves the purpose to link the identified key functions of the natural phenomenon with similar product functionalities. The key subfunctions of the dandelion flower that ignited the idea of a particular product design are: support of multiple individual florets done by the flower head, easy detachment of the seed accomplished by loose joints between the seed and the flower head and flying of the seed in the wind made possible by the special geometry of the pappus. These subfunctions have the same parent-function - seed dispersion and are all connected with the structural aspect of the dandelion. The subfunctions are conducted by the following special subsystems of the dandelion:

- Flower head. Complex, oval, disk-like shape filed with tiny holes that follow a symmetrical pattern and have equal spacing between them. Each hole has conical shape.

- Seeds. A close view reveals that each seed is shaped like a beak at one end. The seed attaches to the flower head via the beak, a thin stalk. The perfect design of the holes on the head section and their difference in diameters make an ideal opening that holds the beak of the seed in position.

- Pappus. The fibrous pappi of the dandelion form a radial array around a central plate. Each dandelion seed contains around 113 pappi with an open angle of about 160-180 degrees [11]. Those pappi make the flight of the seed possible by a parachute mechanism that increases aerodynamic drag. The air rushing between the spaces of the bristles results with a lift-generating vortex that emerges on top of the pappus and enables lift, resembling the parachute-like-drag-increasing mechanism [12].

The functional analysis is illustrated on Figure 2.

\section{Six-step Bionic Strategy}

Following the functional analysis that helps to highlight key bionic functions which could be used in a product design, is the six-step bionic strategy. The six steps allow the designer to understand how the bionic functionalities could become functionalities of a product and to use them as an inspiration in the product concept development phase.

The first step is to analyze in detail the description of the natural phenomenon done in the bionic inspiration overview and the functional decomposition in order for possible applications in product concept generation to become clear.

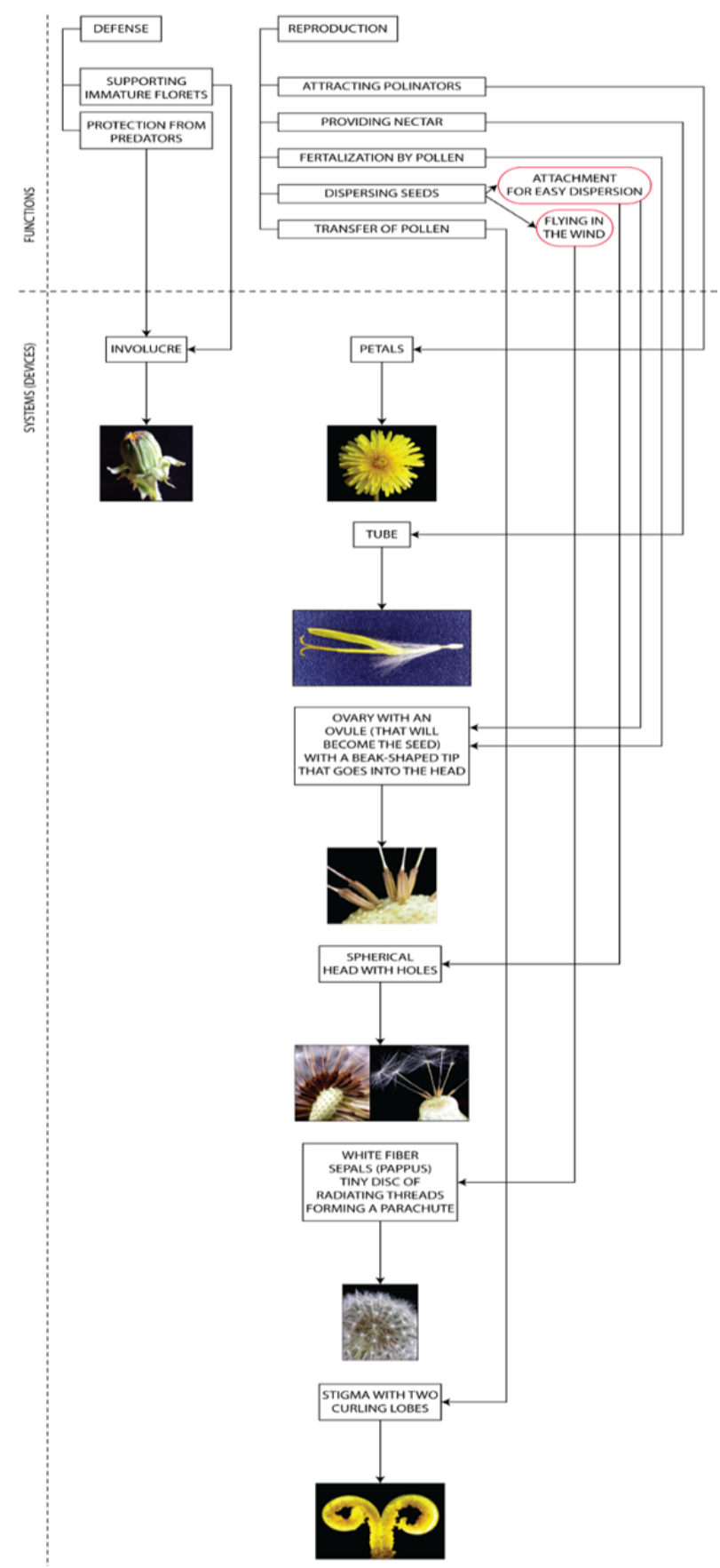

Figure 2. Functional analysis of the dandelion flower (source of images: Brian Johnston, A Close-up View of the Wildflower "Dandelion", microscopy-uk.org.uk/, 2010)

The second step is explaining the natural solution using a "What/Why/How?" tabular presentation. Designers ask themselves "What?" the natural system achieves, "Why?" it conducts those activities and "How?" it makes that possible. This systematized, 
structural representation of the identified natural phenomenon makes it comparable with design problems. The tabular explanation of the natural phenomenon for the dandelion flower is provided in Table 1 .

The third step is redefining the bionic functions and using them to conduct research for particular issues that need to be solved, existing design challenges, novel technologies and trends etc. with the purpose to withdraw design possibilities that could be connected with the bio-functions. To reach this goal, during the research process, the questions: "What needs to be done and why?" and "What can the bio-functionality solve?" are asked. The redefined functions of the dandelion are given in Table 2.

The following steps (step 4 and 5) firstly include an organization of the gathered information for the identified product that could be designed by the principles of the natural solution and then an explanation regarding how that will be achieved. This is done by using the three-column "What/Why/How?" table, but this time for explaining the key product functions. The first column explains what functionalities the product needs to achieve, the second column explains why those functionalities are needed, and the third one describes how they can be achieved by using the previously redefined bionic functions. In the design case of the dandelion flower, the identified product is a street light sculpture with solar panels that needs to have a modular design with a simple joint system for easy assembly, disassembly and repair and needs to have a lightweight support for the solar panels in order to save material and again, be in favor of the modularity function. These functions can be accomplished by designing the joints similarly to the seed-head of flower attachment that the dandelion flower uses and by designing a construction for supporting the solar panels similarly to the structure of the flying pappus. This description is provided in Table 3.

The sixth and final step is the product concept generation and selection. The bio-inspired street lamp was designed as a sculpture with an ambient led light that uses renewable energy sources - solar panels. The appearance of the street light resembles the structure of the dandelion flower (Figure 3). In the center there is a perforated metal sphere (Figure 4). The metal bars that hold the solar panels are mounted in those holes (Figure 5). The bars have a conical rubber at the end, similarly to the beak of the dandelion seeds. This helps seal the joint and, in combination with safety pins, makes the assembly and disassembly process very simple (Figure 6). The bars at the other end have radially placed metal bars with a smaller diameter resembling the distribution of the pappi. Those radial bars hold the lights in the center (Figure 7) and a metal ring on top on which the solar panel is fixed (Figure 8). Overall, the structure is unique, modular, adaptive and lightweight.
Table 1. Page layout description

\begin{tabular}{|l|l|l|}
\hline What? & Why? & How? \\
\hline $\begin{array}{l}\text { Loose joint } \\
\text { system } \\
\text { between the } \\
\text { seed and the } \\
\text { flower head }\end{array}$ & $\begin{array}{l}\text { Easy } \\
\text { removal } \\
\text { when the } \\
\text { seed is ripe }\end{array}$ & $\begin{array}{l}\text { Conical holes of the flower } \\
\text { head and beak tip of the seed }\end{array}$ \\
\hline $\begin{array}{l}\text { Flying of the } \\
\text { seed in the } \\
\text { wind }\end{array}$ & $\begin{array}{l}\text { Dispersion } \\
\text { of the seeds }\end{array}$ & $\begin{array}{l}\text { Geometry of the pappus, } \\
\text { containing around 100 pappi } \\
\text { (with a radial array and an } \\
\text { opengle of about 160-180 } \\
\text { degrees that allow the air to } \\
\text { flow between them and create } \\
\text { a low-pressure vortex which } \\
\text { travels above the pappus and } \\
\text { generates lift }\end{array}$ \\
\hline
\end{tabular}

Table 2. Page layout description

\begin{tabular}{|l|l|}
\hline $\begin{array}{c}\text { What needs to be done and } \\
\text { why? }\end{array}$ & \multicolumn{1}{|c|}{$\begin{array}{c}\text { What can the bio- } \\
\text { functionality solve? }\end{array}$} \\
\hline $\begin{array}{l}\text { Apply the joint system of the } \\
\text { seed with the dandelion head } \\
\text { to provide easy } \\
\text { attachment/detachment }\end{array}$ & $\begin{array}{l}\text { Improve the modularity of a } \\
\text { product for ease of } \\
\text { manufacture, repair and } \\
\text { adaptability }\end{array}$ \\
\hline $\begin{array}{l}\text { Apply the pappus geometry } \\
\text { to reduce the weight }\end{array}$ & $\begin{array}{l}\text { Improve the aesthetics of a } \\
\text { product by using the radial } \\
\text { array to create a unique } \\
\text { design whilst keeping the } \\
\text { product lightweight }\end{array}$ \\
\hline
\end{tabular}

Table 3. Page layout description

\begin{tabular}{|l|l|l|}
\hline \multicolumn{1}{|c|}{ What? } & \multicolumn{1}{|c|}{ Why? } & \multicolumn{1}{c|}{ How? } \\
\hline $\begin{array}{l}\text { Simple joint } \\
\text { system of the } \\
\text { solar panel } \\
\text { holders }\end{array}$ & $\begin{array}{l}\text { Easy } \\
\text { assembly } \\
\text { and repair }\end{array}$ & $\begin{array}{l}\text { Conical holes on a central } \\
\text { sphere and beak-like tip of the } \\
\text { bars that are the body of the } \\
\text { construction which holds the } \\
\text { solar panels }\end{array}$ \\
\hline $\begin{array}{l}\text { Lightweight } \\
\text { system for } \\
\text { supporting } \\
\text { multiple } \\
\text { solar panels }\end{array}$ & $\begin{array}{l}\text { Saving } \\
\text { material }\end{array}$ & $\begin{array}{l}\text { Radially positioned bars at an } \\
\text { angle of 160 degrees placed on } \\
\text { one end of the body of the } \\
\text { construction that hold the solar } \\
\text { panels }\end{array}$ \\
\hline
\end{tabular}

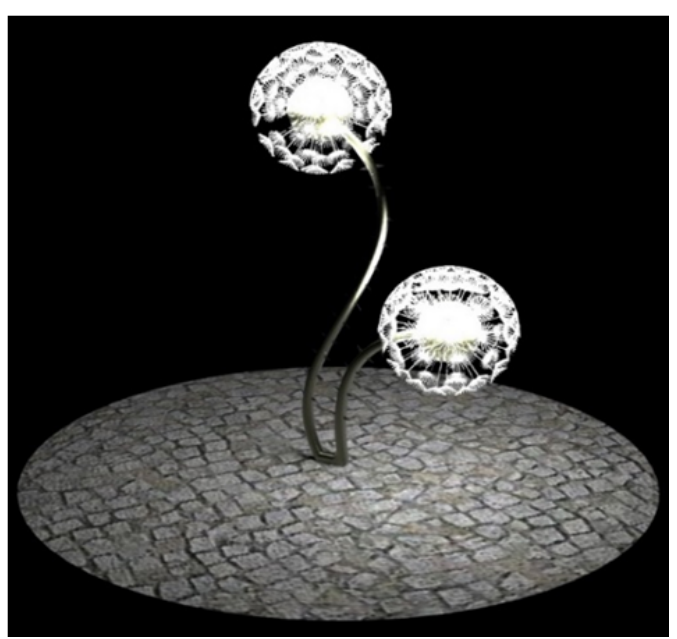

Figure 3. Street lamp sculpture with ambient led light bioinspired from the dandelion flower 


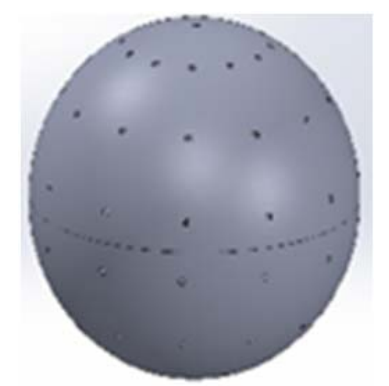

Figure 4. Spherical center with holes

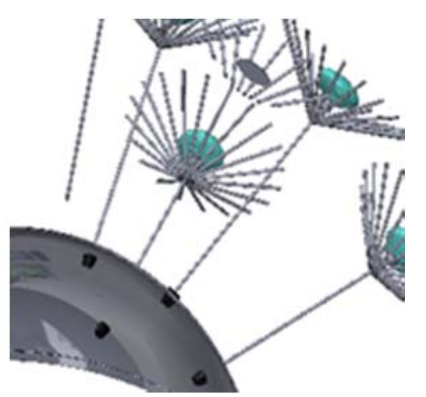

Figure 5. Metal bars with a rubber tip (body of the panelholding structure) inserted in the holes of the central sphere

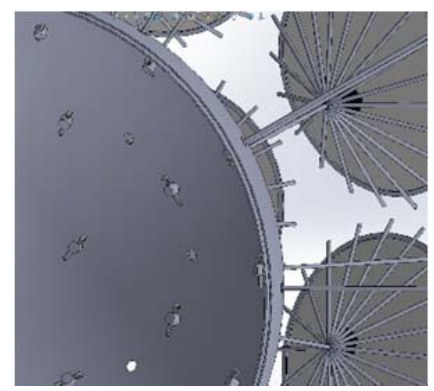

Figure 6. Close-up of the joints between the panelholding structure and the central sphere with safety pins for locking

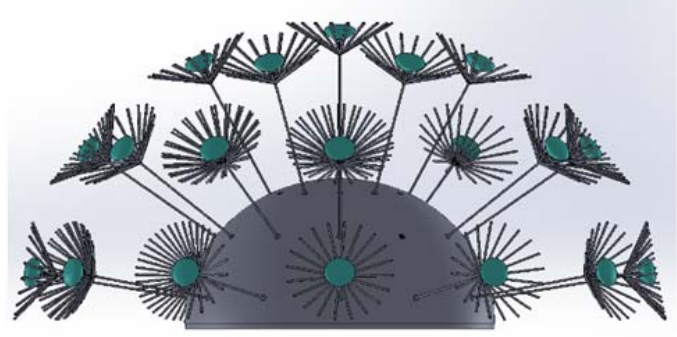

Figure 7. Radially placed bars with a light in the center

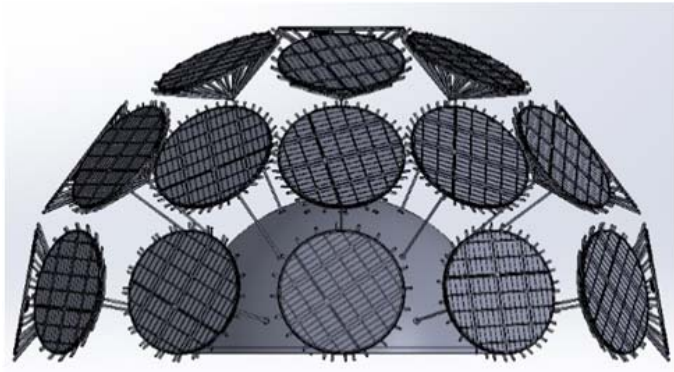

Figure 8. Solar panels mounted on the structure for each lead light

\section{Discussion}

The presented design case study shows the strength of the improved six-step strategy which is the neat systematization of the gathered bionic information. The analysis of the natural phenomenon and the suggested approach for identification of key functions and subsystems as well as their redefinition in a design and engineering context helps not only to achieve a unique design solution, but also to provide a bio-inspired answer, expressed in a technical and directly applicable manner, for improving product functionalities and solving product improvement contradictions. In the case of the solar street light, its joints and structure are bio-inspired by the dandelion flower and are based on the engineering principle of segmentation which helps to solve issues like ease of manufacture, ease of operation, ease of repair, adaptability and device complexity. These redesigned bio-functionalities can be used in other projects where similar solutions are needed. That is in fact the main goal of using this method, to establish a base of tested product functions which were inspired by natural phenomenon and solve particular issues. Designers can go through the base of offered biosolutions whenever they are designing a new product and need inspiration for creating devices that need to conduct particular functionalities. Searching this base can allow them to make different combinations of redesigned bio-functions. This base can be extended each time a new natural phenomenon catches the attention of the designers and is used in a project.

\section{Conclusion}

This paper elaborates an improved six-step strategy for bionic inspiration in industrial design by using a design case study. The inspiration for the described design solutions came from the dandelion flower and its unique characteristics and functionalities. The explanation of this natural phenomenon, the conducted functional analysis and the definition of components that make those functionalities possible was the base for researching design areas where those functionalities can be used in order to improve particular design features. The chosen product for design was a street led lamp with solar panels. The key bionic functions identified "simple and easily detachable joints between the seed and the flower head" and the "unique structure of the pappus for seed dispersion" were redefined in order to make them applicable in the process of creating suitable design solutions for the street lamp. The end result was a unique light sculpture with an appearance similar to the dandelion which catches the attention of passers-by and in addition, is easy for production, mounting and repair due to the modular 
system (based on the joints identified in the dandelion) which is simple and does not add unnecessary weight to the whole structure.

The case study explained, as well as other related studies [13], prove the success of the proposed sixstep strategy for seeking bionic inspiration, solving contradictions and creating a base of redefined bionic principles that answer particular design problematics. However, further research should include developing a product prototype (for example by additive manufacturing) and its evaluation for improvement of the structure and investigating whether the bionic principles were applied in a correct manner.

\section{References}

[1]. Benyus, J. M. (1997). Biomimicry: Innovation inspired by nature (p. 320). New York: Morrow.

[2]. Versos, C. A., \& Coelho, D. A. (2013). A BiDirectional Method for Bionic Design with Examples. In Advances in Industrial Design Engineering. IntechOpen.

[3]. Helms, M., Vattam, S. S., \& Goel, A. K. (2009). Biologically inspired design: process and products. Design studies, 30(5), 606-622.

[4]. Goel, A. K. (2015, June). Is Biologically Inspired Invention Different?. In ICCC (pp. 47-54).

[5]. Stevanović, I., \& Rašuo, B. (2017). Development of a miniature robot based on experience inspired by nature. FME Transactions, 45(1), 189-197.

[6]. Filippov, A. E., Gorb, S. N., \& Popov, V. L. (2015). What Can we Learn from the "Water Bears" for the Adhesion Systems Using in Space Applications?. Facta Universitatis, Series: Mechanical Engineering, 13(3), 241-247.

[7]. Coelho, D. A., \& Versos, C. A. (2011). A comparative analysis of six bionic design methods. International Journal of Design Engineering, 4(2), 114-131.

[8]. Santos-Reyes, D. E., \& Lawlor-Wright, T. (2009). Understanding design in nature can benefit product conceptual design. International Journal of Design \& Nature and Ecodynamics, 4(2), 105-122.

[9]. Forniés, I. L., \& Muro, L. B. (2012). A top-down biomimetic design process for product concept generation. International Journal of Design \& Nature and Ecodynamics, 7(1), 27-48.

[10]. Han, Y. L., Li, M., Yang, Q., Huang, G., Liu, H., Qin, Y., ... \& Xu, F. (2017). Collective Wetting of a Natural Fibrous System and Its Application in PumpFree Droplet Transfer. Advanced Functional Materials, 27(22), 1606607.

[11]. Meng, Q., Wang, Q., Liu, H., \& Jiang, L. (2014). A bio-inspired flexible fiber array with an open radial geometry for highly efficient liquid transfer. NPG Asia Materials, 6(9), e125-e125.

[12]. Cummins, C., Seale, M., Macente, A., Certini, D., Mastropaolo, E., Viola, I. M., \& Nakayama, N. (2018). A separated vortex ring underlies the flight of the dandelion. Nature, 562(7727), 414-418

[13]. Angeleska, E., \& Sidorenko, S. (2021). Bio-inspired back support system for backpacks. FME Transactions, 49(2), 327-334. 